\title{
Study of Solutions of Poly(dimethylsiloxane) in Benzene by NMR
}

\author{
Akihiro Muramoto \\ Material Research Laboratory, Toshiba Research and Development Center, \\ Kawasaki, Kanagawa, Japan.
}

(Received December 6, 1973)

\begin{abstract}
Solutions of poly(dimethylsiloxane) in benzene have been studied by the analysis of NMR spectra. It was found that they are characterized by the narrow-broad lines previously obtained for pure liquid poly(dimethylsiloxane). The width and the second moment of the absorption bands for the methyl group and for benzene varied almost in the same manner with decreasing volume fraction of poly(dimethylsiloxane) and showed a strong dependence on volume fraction of poly(dimethylsiloxane) in highly concentrated solution above the critical entanglement point of the chains. These trends suggest that the correlation time for the nuclear magnetic interaction of benzene as well as for the methyl group are governed by the segmental motion and the drift motion of the poly(dimethylsiloxane) molecules as a whole. In the highly concentrated solution, a sharp peak due to the increase of the isolated benzene molecules superposes on the so-called narrow-broad line, which is attributed to the benzene molecules interacting with the poly(dimethylsiloxane) molecules. Thus the ratio of $\Delta H_{1 / 2}$ to $2 \sqrt{\left\langle\Delta H_{2}{ }^{2}\right\rangle}$ for benzene decreases.
\end{abstract}

KEY WORDS Poly(dimethylsiloxane) / Solution / Benzene /NMR / Relaxation / Second Moment / Viscosity / Molecular Motion /

The line shape of the NMR spectrum for poly(dimethylsiloxane) has been studied by Rochow, ${ }^{1}$ Kusumoto, ${ }^{2}$ and others. Since poly(dimethylsiloxane) contains only one type of proton, line-broadening of the spectrum does not arise from nuclear magnetic cross-relaxation and spin coupling. It has been proved that the methyl group rotates about the $\mathrm{Si}-\mathrm{C}$ bond with unusual freedom; ${ }^{1}$ at higher temperatures, the intramolecular spiral motion of the dimethylsilyl group ${ }^{2}$ and the segmental motion of the molecule as a whole ${ }^{3}$ are added to the motion of the magnetic protons in the methyl group. It is naturally expected that such motions are more intensive if the poly(dimethylsiloxane) molecules are in a solvent, rather than being kept at a higher temperature.

The nuclear magnetic relaxation in a solution of chain macromolecules has been analysed in terms of the relative motion of the polymer segment containing magnetic nuclei on the basis of the Brownian motion. ${ }^{4}$ Poly(dimethylsiloxane) in solution shows two types of molecular motion: one is the segmental motion of the chain due to the effect of thermal transitions, and the other is the drift motion of the chain as a whole. In this polymer, the spin-lattice relaxation time depends on the local, intrachain nuclear magnetic interaction and is independent of the change of viscosity, on which the spin-spin relaxation time depends. ${ }^{5}$ On the other hand, the spinspin relaxation time is governed chiefly by the drift motion of the chain and rather mildly by the internuclear magnetic interaction. Thus, the absorption curve shows a so-called narrow-broad line $^{6}$ and the spin-spin relaxation time significantly depends on viscosity. ${ }^{5}$ The critical entanglement point of macromolecular chains ${ }^{7}$ has been found at about 30,000 of molecular weight, ${ }^{8}$ where a break point appears on the curve representing the relation between viscosity and molecular weight. The entanglement of poly(dimethylsiloxane) molecules influences the dependence of the self-diffusion coefficient on concentration. ${ }^{9}$

The NMR spectra of benzene in poly(dimethylsiloxane) solution can be clearly distinguished from those of the methyl group in poly(dimethylsiloxane) because of the large difference in their chemical shifts. Theories for various inter- and 
intramolecular magnetic nuclear dipole-dipole interactions for benzene are presented in the literature. ${ }^{10-13}$ There are two mechanisms in the relaxation of the nuclear magnetization for benzene. ${ }^{10}$ One is the direct nuclear magnetic dipole-dipole interaction, that gives rise to relaxation because of the spatial reorientation and transition of the molecules. The other is the spin-rotation interaction, which slightly contributes to the spin-lattice relaxation time of the proton of benzene even at room temperature. $^{10}$

The width or the second moment for the solvent in a polymer solution depends on the concentration; this makes it possible to analyse the motion of the solvent molecules and the interaction of the solvent with the polymer in the solution. Odajima has found by an NMR analysis method that the water absorbed on native cellulose does not rotate in spherical symmetry, as occurs in the liquid state. ${ }^{14}$

In this report, the half width and the second moment of NMR spectra for the methyl group and benzene were measured in poly(dimethylsiloxane)-benzene mixtures by a high-resolution NMR apparatus. The results obtained were analysed in order to investigate the formation of a solution through mixing poly(dimethylsiloxane) with benzene.

\section{THEORETICAL}

An interpretation of experimental results for the nuclear-magnetic-resonance absorption can be made, based on present theories as follows.

The spin-spin relaxation time for polymer molecules in solution has been calculated on the basis of the relative motion of the segment of a polymer containing nuclei which itself undergoes a complicated Brownian motion, in terms of the Zimm-Rouse model ${ }^{15}$ (also known as the Kargin-Slonimskii model $^{16}$ ) for chain macromolecules as follows ${ }^{17}$

$$
\left(T_{2}\right)^{-1}=\sigma^{2} C_{(0)}+\sigma^{2}\left[\frac{5}{3} C_{\left(\omega_{0}\right)}+\frac{2}{3} C_{\left(2 \omega_{0}\right)}\right]
$$

where $\sigma^{2}$ is the total second moment, and the first and the second terms give the adiabatic and the nonadiabatic contribution to the spinspin relaxation time, respectively. $C_{(0)}$ and $C_{\left(\omega_{0}\right)}$ for molecules of high molecular weight have been approximately calculated to be

$$
C_{(0)}=1.2 \ln n, \quad C_{\left(\omega_{0}\right)}=0.6 \ln \left(1.4 / \tau \omega_{0}\right)
$$

where $\tau$ is the correlation time and $n$ is the number of segments of a chain.

The nuclear-spin system observed in poly(dimethylsiloxane) in solution is strongly fluctuating. For such a system, the adiabatic contribution to the spin-spin relaxation time, $T_{2 \mathrm{~s}}^{*}$, is observed alone by a high-resolution NMR apparatus, using the following equation. ${ }^{18}$

$$
\left(T_{2 \mathrm{~s}}^{*}\right)^{-1}=(3 / 10) \sigma_{\mathrm{s}}{ }^{2} C_{(0)}
$$

The magnetic nuclei on poly(dimethylsiloxane) in solution undergo three modes of motion: (1) the rotation of the methyl group about the bond of $\mathrm{Si}-\mathrm{C},(2)$ the extraordinary free rotation of the dimethylsillyl group about the bond of $\mathrm{Si}-\mathrm{O},{ }^{3}$ and $(3)$ the anisotropic reorientation in which a spin pair reorients randomly about the axis which, in turn, tumbles randomly. ${ }^{4}$ The main contribution to the relaxation process is the interaction between protons on the same methyl group, since the distance between a pair of protons on different methyl groups is very large. Thus, the relaxation time for poly(dimethylsiloxane) in solution can be considered to be determined by a rotation of the internuclear vector about an axis which, during the same time, undergoes a random reorientation at different rate.

The theory of spin relaxation developed by Woessner ${ }^{20}$ may be applied for such a relaxation process. On the assumptions that the rotation about the axis occurs in random jumps between three equilibrium positions $120^{\circ}$ apart at an average rate of $\left(3 \tau_{\mathrm{c}}\right)^{-1}$, while the correlation time for the reorientation of the rotation axis is $\tau_{\mathrm{c} 1}$, eq 3 is rewritten as

$$
\begin{aligned}
\left(T_{2 \mathrm{~S}}^{*}\right)^{-1}= & (3 / 10){\sigma_{\mathrm{s}}}^{2}\left[\frac{1}{4}\left(3 \cos ^{2} \Delta-1\right)^{2} \tau_{\mathrm{c} 1}\right. \\
& \left.+\frac{3}{4} \sin ^{2} 2 \Delta \tau_{\mathrm{c} 2}+\frac{3}{4} \sin ^{4} \Delta \tau_{\mathrm{c} 3}\right]
\end{aligned}
$$

where $\tau_{\mathrm{c} 2}$ and $\tau_{\mathrm{c} 3}$ are given as follows

$$
\tau_{\mathrm{c} 2}=\left(\frac{1}{\tau_{\mathrm{c} 1}}+\frac{1}{\tau_{\mathrm{c}}}\right)^{-1}, \quad \tau_{\mathrm{c} 3}=\left(\frac{1}{\tau_{\mathrm{c} 1}}+\frac{4}{\tau_{\mathrm{c}}}\right)^{-1}
$$

and $\Delta$ is the angle between the internuclear vector and the rotation axis. The average angles $\bar{\Delta}$ for three interproton vectors have been given 


\section{A. Muramoto}

by $0^{\circ}, 60^{\circ}$, and $120^{\circ} .^{21}$ If the system is characterized by a wide variety of relaxation times and the relaxation process may present the same behavior as the multiphase system does, the spinspin relaxation time becomes ${ }^{22}$

$$
\left(T_{2 \mathrm{~s}}^{*}\right)^{-1}=\sum_{i} P_{i}\left(\tau_{i}\right)\left(T_{2 \mathrm{~s}}^{*}\right)^{-1}\left(\tau_{i}\right)
$$

where $P_{i}$ is the fraction of nuclei in molecules with jump time $\tau_{i}$ and $\sum P_{i}=1$. If the phase exchange of nuclei takes place very rapidly, a single relaxation time is $\operatorname{seen}^{22}$ :

$$
\left(T_{2 \mathrm{~b}}^{*}\right)^{-1}=\sum_{i} P_{i}\left(T_{2 i}^{*}\right)^{-1}
$$

where $P_{i}$ is the fraction of nuclei in molecules with relaxation time $T_{2 i}$. For slow exchange the system represents as many relaxation times as there are phases.

On the other hand, the lattice system of benzene in a polymer solution is strongly fluctuating, so that the spin-spin relaxation time for benzene is given as

$$
\left(T_{2 \mathrm{~b}}\right)^{-1}={\sigma_{\mathrm{b}}}^{2} \tau_{\mathrm{b}}
$$

where ${\sigma_{\mathrm{b}}}^{2}$ is the total second moment and $\tau_{\mathrm{b}}$ is the correlation time for the Brownian motion of benzene molecules. In a concentrated solution of polymer, however, environments for the benzene molecules are microscopically inhomogeneous. Such a system is characterized by a very broad distribution of correlation times, which leads to an apparent phase transition in the spin-spin relaxation time. ${ }^{23}$ Therefore, the observed $T_{2 \mathrm{~b}}$ can be interpreted as one aspect of the microscopic inhomogeneity of the viscosity in the polymer solution.

\section{EXPERIMENTAL}

Poly(dimethylsiloxane) was synthesized by the ordinary method and was treated to cut off a low molecular portion by precipitation in methyl alcohol from tetrachloromethane solution. From the intrinsic viscosity, ${ }^{24}$ the molecular weight was found to be 220,000 . Benzene used in these experiments was purified by distillation from spectroscopic grade reagent. Polymer solutions were prepared beforehand by mixing a known amount of solvent and polymer in the range of volume fraction of polymer from 0.036 to 0.66 . Less
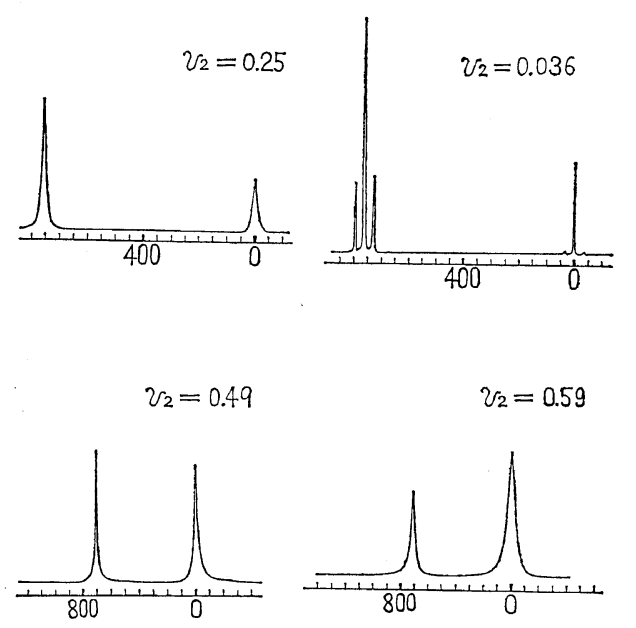

Figure 1. Representative NMR spectra of solutions of poly(dimethylsiloxane) in benzene at various concentrations, in $\mathrm{Hz}$.

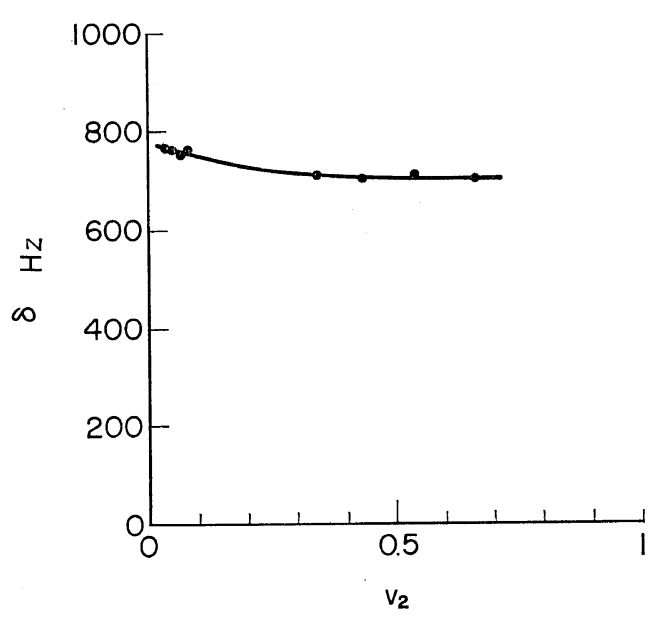

Figure 2. Variation of chemical shift with concentration for benzene in the spectrum of the methyl group in poly(dimethylsiloxane)-benzene mixtures.

viscous solutions were poured into the NMR sample tubes. Highly viscous solutions above about 40-percent concentration were thoroughly frozen with dry ice and acetone, and small lumps of the frozen solution were brought into the NMR sample tubes. All sample tubes were then sealed and kept at room temperature for several days to insure uniform dissolving.

Measurements of proton NMR spectra were 
Study of PDS-Benzene Solutions by NMR

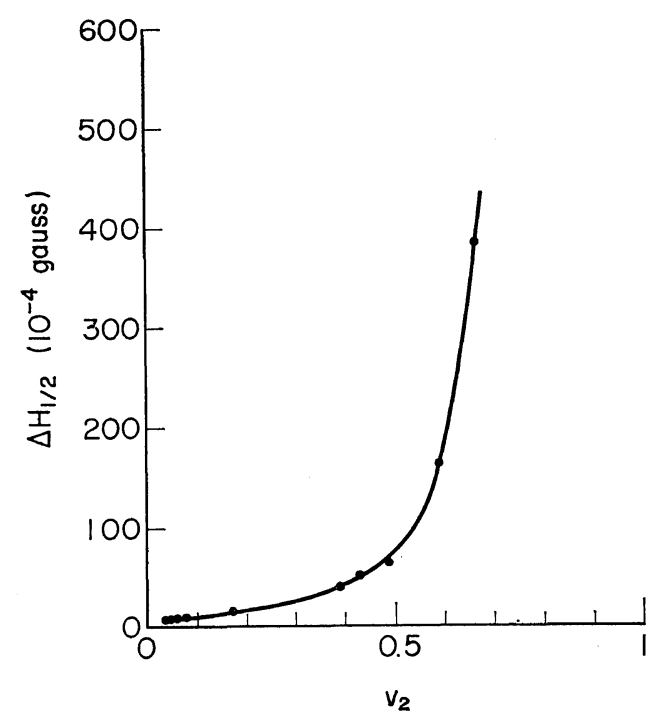

Figure 3. Variation of the half width with concentration for the methyl group in poly(dimethylsiloxane)-benzene mixtures.

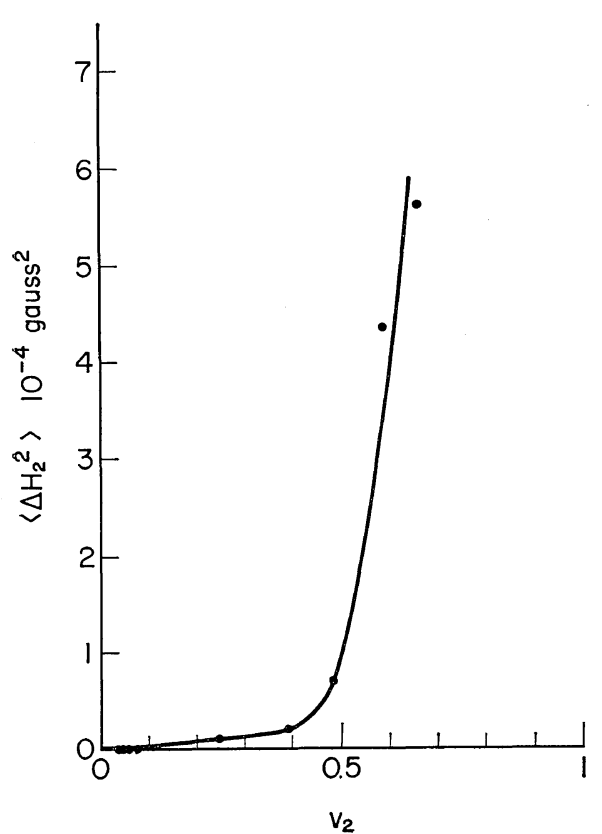

Figure 4. Variation of the second moment with concentration for the methyl group in poly(dimethylsiloxane)-benzene mixtures.

made by using a high-resolution spectrometer at a central frequency of $100 \mathrm{MHz}$ and at room temperature, about $20^{\circ}$. The spectra obtained

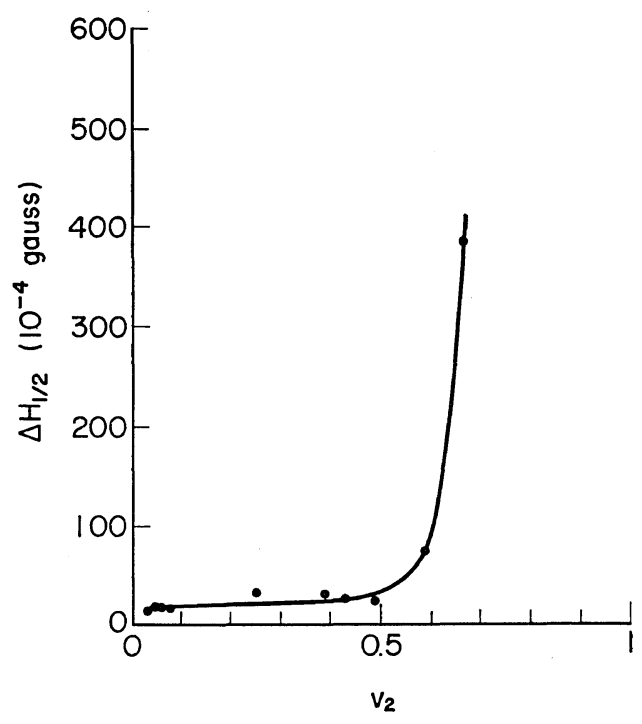

Figure 5. Variation of the half width with concentration for benzene in poly(dimethylsiloxane)benzene mixtures.

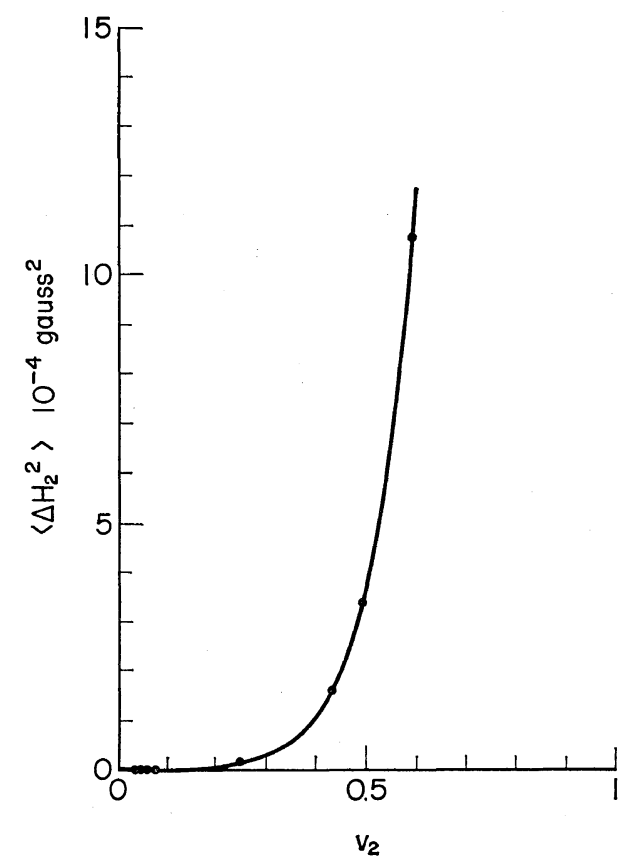

Figure 6. Variation with concentration of the NMR spectra for benzene in poly(dimethylsiloxane)-benzene mixtures.

for the methyl group and benzene are shown in Figure 1. The chemical shifts for the protons of the methyl group could not be accurately 


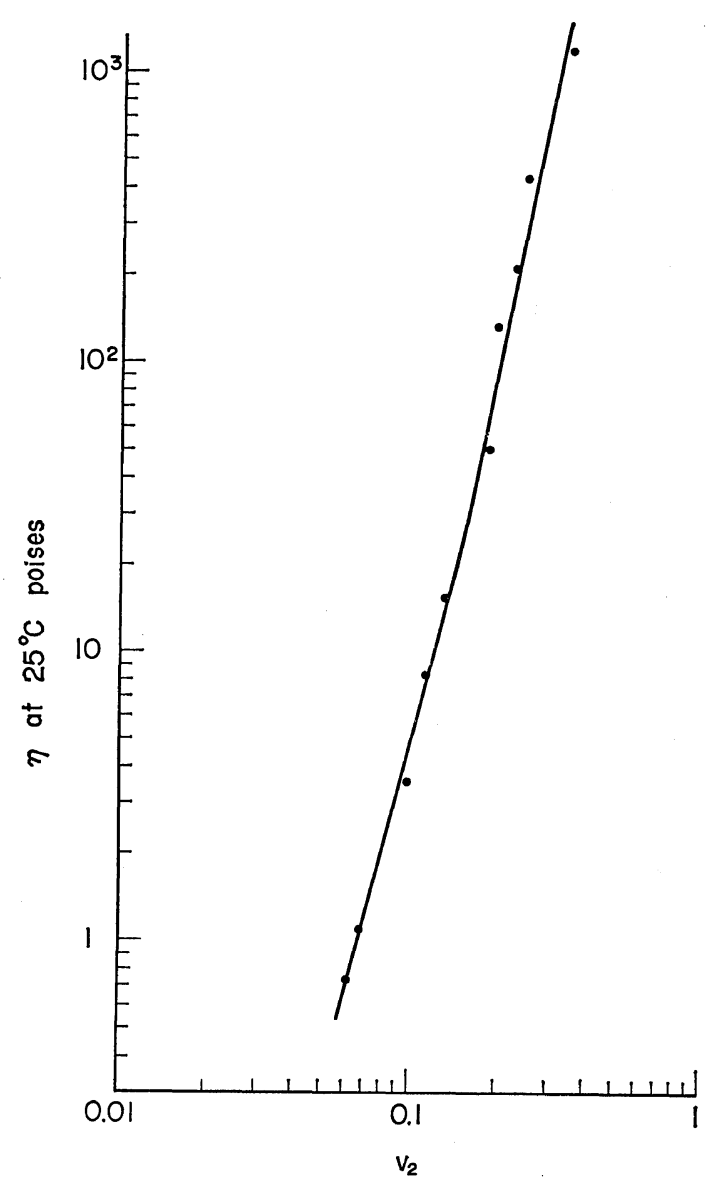

Figure 7. Dedendence of the viscosity of solutions of poly(dimethylsiloxane) in benzene on concentration at $25^{\circ} \mathrm{C}$.

measured because the reference spectrum of tetramethylsilane superposed upon the spectrum of methyl group. In this experiment, the difference of chemical shifts between the spectra of the methyl group and benzene is represented in hertz $(\mathrm{Hz})$ in Figure 2. The half width and the second moment are plotted against the volume fraction $v_{2}$ of polymer in Figures 3, 4, 5, and 6.

The viscosities of the above-mentioned solutions were measured using a rotational viscometer at $25^{\circ} \mathrm{C}$. In Figure 7 , the viscosity is plotted against $v_{2}$.

\section{RESULTS AND DISCUSSION}

The spectra obtained in this study (as shown in Figure 1) were single peaks, in agreement with the previous report. ${ }^{19}$ The spectrum for benzene in the dilute solution may contain two peaks in addition to the central peak. These side peaks were observed only at this concentration studied and are not assigned clearly, but conceivably originate from fluctuations of the local polymer concentration around the benzene molecules. ${ }^{25}$ On the other hand, the single peak for the methyl group obtained in this experiment is attributed to the peak characteristic of highmolecular-weight poly(dimethylsiloxane).

Many conformations are available to the poly(dimethylsiloxane) chain and the chain dimension in concentrated solution may be approximated to that of the unperturbed chain in dilute solution. ${ }^{26}$ The configurational statistics of the chain suggests that the planer trans conformation is preferable for poly(dimethylsiloxane). ${ }^{27}$ The angle $\mathrm{O}-\mathrm{Si}-\mathrm{O}$ in the case of the unstrained molecule is assumed to be $110^{\circ}$ for tetrahedral angle, ${ }^{28}$ but the angle $\mathrm{Si}-\mathrm{O}-\mathrm{Si}$ is easily varied by the translational motion of the molecule. Flory and coworkers ${ }^{29}$ obtained $143^{\circ}$ as the largest possible value of the angle $\mathrm{Si}-\mathrm{O}-\mathrm{Si}$. In the trans conformation the neighbor pair of methyl groups on the same side of the plane are favorably $3.78 \AA$ apart from one another. Those on the opposite sides of the plane are $4.88 \AA$ apart, which is beyond the range of significant interaction. Therefore the nuclear magnetic relaxation of the methyl group is completely dominated by the former interaction, since the distance between a pair of hydrogen atoms on the same methyl group is much shorter than the distance between hydrogen atoms on different methyl groups. ${ }^{30}$ On the other hand, taking the values $1.39 \AA$ and $1.08 \AA$ for the $\mathrm{C}-\mathrm{C}$ and the $\mathrm{C}-\mathrm{H}$ bond lengths respectively, the radius of the benzene molecules (distance between $\mathrm{H}-\mathrm{H}$ on diagonal line) is $4.94 \AA ;^{10}$ this value is rather larger than the distance between neighboring methyl groups.

The difference, $\delta$, in chemical shift between the peaks for the methyl group and the peak for benzene is shown as a function of $v_{2}$ in Figure 2. That is, $\delta$ is almost constant except for the extreme dilute solution range, and slightly increases as the concentration of the solution decreases. The chemical shift for a polymer in benzene depends on its solubility in the solvent. ${ }^{31}$ 
The more strongly the polymer molecules interact with benzene molecules in solution, at the higher magnetic field the nuclear spin in the polymer resonates due to the effect of the ringcurrent. ${ }^{32}$ It is found in the dilute solution that poly(dimethylsiloxane) shows a tendency toward strong interactions with benzene. ${ }^{33,34}$ Accordingly, it is conceivable that the chemical shift for the methyl group decreased in dilute benzene solution.

Poly(dimethylsiloxane) contains one type of proton having two near-neighbors. It may be speculated, therefore, that the local internuclear magnetic field acting on chains of the polymer reveals the dominant relaxation process. This implies that the nuclear magnetic relaxation takes place as a consequence of both the Brownian motion of the polymer chains and the relative motion of the spins with respect to the polymer segment to which they are attached. ${ }^{4}$ The radius of molecular motion of a polymer coil in solution depends mainly on the polymer segmentsolvent interaction, which increases with decreasing concentration. ${ }^{35}$ Therefore, the shape of spectrum is closely related to the concentration.

A methyl group of poly(dimethylsiloxane) attaches to the $\mathrm{Si}$ atom which, in turn, is bound to two oxygen atoms. Woessner's equation, which is formulated with correlation times for the rotation of the methyl group and for the reorientation of the rotation axis, may be applied to analyse the nuclear magnetic relaxation of poly(dimethylsiloxane) in solution. ${ }^{21}$ The correlation time for the $\mathrm{CH}_{3}$ rotation is characterized by a single rate constant, since this rotational motion takes place independently of the cooperative motion of the rest of chain molecules. On the other hand, the correlation time of the reorientation of the rotation axis is presumably affected by correlated movements between adjacent segments.

The viscosity is expressed ${ }^{36}$ by the product of the reciprocal of the segmental jump frequency, $J$, and the correlation factor, $F$, for the motion of the segment.

$$
\eta=F / J
$$

The segmental jump frequency depends on the temperature and free volume for segmental flow for a given substance. Accordingly, it obviously depends on the bulk viscosity. ${ }^{5}$ While it has been found in the solution of poly(dimethylsiloxane) in tetrachloroethylene that $\tau_{\mathrm{r}}$ is proportional to $\eta^{1 / 2}$ when $\eta<\eta_{c}$, but to $\eta^{1 / 6}$ when $\eta>\eta_{\mathrm{c}},{ }^{21}$ since the spin-spin relaxation time of the methyl group for poly(dimethylsiloxane) is not changed with the kind of solvent in the solution, ${ }^{21}$ the relation between the viscosity and $\tau_{\mathrm{r}}$ mentioned above is applicable for the solution of poly(dimethylsiloxane) in benzene. It may be concluded that the half width and the second moment for the methyl group depend on the viscosity of the solution through the variation of $\tau_{\mathrm{r}}$ with concentration.

It has been found ${ }^{5}$ that the motion as a whole of the molecule of liquid poly(dimethylsiloxane) predominantly determines the spin-spin relaxation time, because the transverse decay naturally depends on the concentration at a given temperature for a given number-average chain length and a given species of a diluent, since the free volume is associated with the local configurational arrangement of the nearest neighbor segments about a given segment. On the other hand, the coordination factor is postulated to be independent of the free volume at a given temperature. Therefore, for a given molecular weight at a fixed temperature, the viscosity of a poly(dimethylsiloxane) solution depends on the concentration through the change in the segmental jump frequency. ${ }^{35}$

The abrupt change in the slope of the concentration dependence of the viscosity appears at the point at which the interchain entanglement becomes possible, because the segmental jump frequency depends on the local conformational arrangement of the nearest neighbor segment. Figure 7 represents the dependence of the solution viscosity on the volume fraction of poly(dimethylsiloxane), $v_{2}$. The critical point is clearly observed at 0.15 of $v_{2}$.

The half width and the second moment observed for methyl groups of poly(dimethylsiloxane) in solution are shown in Figures 3 and 4 as a function of $v_{2}$. Figure 3 illustrates that the half width for the methyl group at low concentration increases gradually with increasing concentration and rises rapidly at concentrations higher than the concentration at the critical point found in the viscosity - concentration relationship in Figure 


\section{A. Muramoto}

7. The second moment for the methyl group shows nearly the same behavior as does the half width in Figure 4.

The second moment for benzene shown in Figure 6 changes with the volume fraction of poly(dimethylsiloxane) in the same way as that for the methyl group does. The second moment decreases rapidly with the increment of the amount of benzene added to the bulk poly(dimethylsiloxane), till the amount of benzene reaches to about 0.86 by volume fraction. In this range, it is much broader than that for pure benzene at the same temperature. This trend suggests that the entanglement of polymer chains is effective in producing the nuclear magnetic relaxation of solvent molecules in the polymer solution, in which the solvent molecules are absorbed on the segments of the polymer. ${ }^{37}$ However, the addition of benzene makes the entangled chains of poly(dimethylsiloxane) free and the benzene molecules are provided with more freedom for their Brownian motion. This leads to a rapid decrease of the width for benzene in dilute solution.

A part of the width for benzene is the width arising from the contribution of the intermolecular nuclear magnetic dipole-dipole interaction, ${ }^{11}$ which, in pure benzene, is estimated to be 30 or 50 percent of the total interaction. ${ }^{13}$ On the other hand, at the temperature investigated here, the spin-rotation relaxation is negligible because the spin-rotation interaction is very weak. ${ }^{10}$ Accordingly, the contribution of spin-rotation to the width for benzene in the polymer solution is small. Therefore, the much broader width for benzene in highly concentrated solutions, as shown in Figures 5 and 6 , is attributed to the broad distribution of the correlation frequencies arising from the locally high viscosity of the solution. ${ }^{23}$

When the entanglement of poly(dimethylsiloxane) chains turns out to be small enough to allow the segments of poly(dimethylsiloxane) free motion, the width of the absorption for benzene no longer shrinks so rapidly with increasing content of benzene. In dilute solutions, the observed width of absorption for benzene is of the same order as the one for pure benzene, which is broadened only by the inhomogeneity of the magnet used. The gradual rise of the

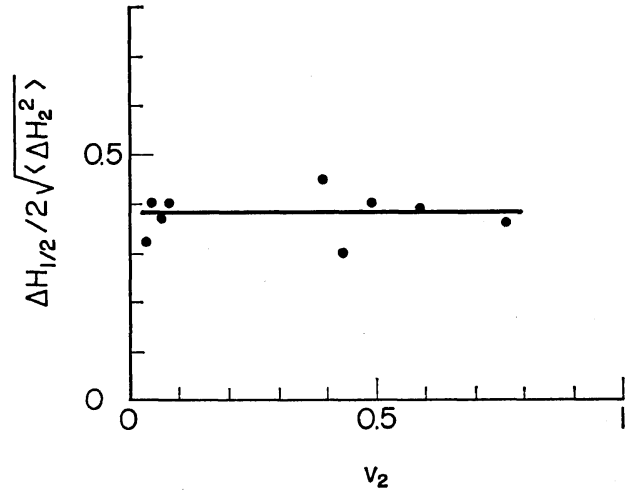

Figure 8. Shape factor, $\Delta H_{1 / 2} / 2 \sqrt{\left\langle\Delta H_{2}^{2}\right\rangle}$, plotted against concentration for the methyl group in poly(dimethylsiloxane)-benzene mixtures.

width with increasing concentration in the range of dilute solution is probably due to the decrease of the correlation frequency of the translational motion, since it depends on the viscosity of the solution.

When the observed absorption spectrum broadens by the adiabatic contribution alone the change in shape of the absorption curve can be seen through the ratio $\Delta H_{1 / 2} / 2 \sqrt{\left\langle\Delta H_{2}^{2}\right\rangle} ;{ }^{38}$ for a Gaussian shape it is unity, for a Lorentzian shape it is infinite. Figure 8 shows a plot of the ratio $\Delta H_{1 / 2} / 2 \sqrt{\left\langle\Delta H_{2}{ }^{2}\right\rangle}$ for the methyl group of poly(dimethylsiloxane) in solution and illustrates that it is constant in the range of concentration studied. That is, the shapes of the absorption curves for the methyl group are unchanged in the range of concentration studied and are not Gaussian, because the constant ratio is 0.38 . This implies that these spectra are the narrowbroad lines named by Powles, ${ }^{6}$ which are only observed when the multiple interactions with different correlation frequencies within the methyl group are substantially motionally averaged. This agrees with the relaxation process observed in bulk poly(dimethylsiloxane) with high molecular weight. ${ }^{5}$

Figure 9 shows a plot of the ratio $\Delta H_{1 / 2}$ l $2 \sqrt{\left\langle\Delta H_{2}^{2}\right\rangle}$ for benzene in poly(dimethylsiloxane) solution against polymer concentration and illustrates the change in shape of the absorption curve with polymer concentration. For pure liquid benzene the absorption curve should be sharply narrowed in a Lorentzian shape under 


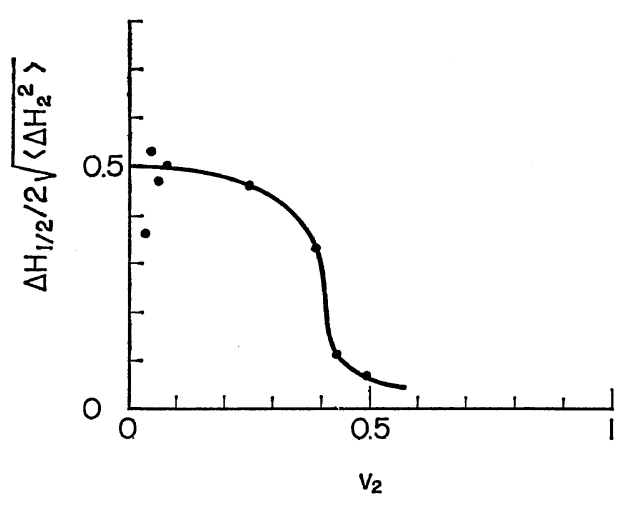

Figure 9. Shape factor, $\Delta H_{1 / 2} / 2 \sqrt{\left\langle\Delta H_{2}{ }^{2}\right\rangle}$, plotted against concentration for benzene in poly(dimethylsiloxane)-benzene mixtures.

the condition of $T_{2}=T_{1}$, because all the correlation times for liquid benzene obey the condition $\tau \ll \omega^{-1}$. The observed spectra for benzene in the dilute solutions, however, remarkably broadens, as shown in Figure 9. It may be considered in this case that the region of the spectrum spreads toward values of the correlation time larger than that of pure liquid benzene and the distribution of the correlation times includes larger values than $\omega^{-1}$, because the reorientation of benzene molecules arises with the cooperation of the segmental motion of poly(dimethylsiloxane) molecules. When a very broad distribution of correlation times characterizes a material, the nuclei can move from one phase to another at various rates. $^{21}$ However, if the exchange is very fast, a single average relaxation time should be found, as in eq $7 .^{21}$ This conclusion elucidates the reason why the absorption lines observed are single and without structure. The same fact has been found in the water absorbed in cellulose; here the spectrum shape is broad even with a large content of water. ${ }^{14}$

In highly concentrated solutions of poly(dimethylsiloxane), the ratio of $\Delta H_{1 / 2}$ to $2 \sqrt{\left\langle\Delta H_{2}{ }^{2}\right\rangle}$ for benzene decreases rapidly, as shown in Figure 9. But the half width and the second moment of these spectra are not so small as those of the Lorentzian curves for low-viscosity liquids. In such a solution, some benzene molecules might be isolated from the poly(dimethylsiloxane) molecules and maintain rather limited Brownian motion. It seems reasonable that such behavior of benzene molecules are responsible for the shape of the spectrum, the half width of which is large relative to $2 \sqrt{\left\langle\Delta H_{2}{ }^{2}\right\rangle}$.

The activity of benzene molecules in the solution of poly(dimethylsiloxane) have been obtained by thermodynamic measurements. ${ }^{33}$ It has been shown that benzene is less compatible to poly(dimethylsiloxane) as the concentration increases and it turns to a poor solvent at $v_{2}=0.7 .^{33}$ It is possible that the above-mentioned change of the shape of spectrum for benzene with concentration corresponds to a decrease in its solubility.

It has been shown that the NMR spectra of poly(dimethylsiloxane) in benzene were subjected to the same principle as was effective for liquid poly(dimethylsiloxane), that is, the width and the second moment for the methyl group could be investigated based on the segmental motion of poly(dimethylsiloxane) molecules. The shape of the NMR absorption for the polymer solution was closely related to the Brownian motion of the polymer molecules. The cooperating variation of the NMR absorption shape for benzene with concentration with that for the methyl group implies that the Brownian motion of benzene molecules in the poly(dimethylsiloxane) solution was dominated by poly(dimethylsiloxane) molecules through thermodynamic interactions between poly(dimethylsiloxane) and benzene molecules. It is noticeable, however, that the width of the NMR spectrum for benzene became small relative to the second moment in highly concentrated solution, in which a part of benzene molecules were isolated from the solution.

Acknowledgement. The author is especially indebted to Professor A. Nakajima of the Department of Polymer Chemistry, Kyoto University, whose elaborate criticism led to considerable improvement in the original manuscript. He would like to express his hearty thanks to Professor S. Fujiwara of the Department of Chemistry, University of Tokyo, for invaluable suggestions on the adaptation of the NMR method to polymer soltions and for measuring the NMR spectra.

\section{REFERENCES}

1. E. G. Rochow and H. G. LeClair, J. Inorg. Chem., 1, 92 (1955). 


\section{A. Muramoto}

2. H. Kusumoto, I. J. Lawrenson, and H.S. Gutowsky, J. Chem. Phys., 32. 724 (1960).

3. W. L. Ruth, J. Amer. Chem. Soc., 69, 474 (1974).

4. R. Ullman, J. Chem. Phys., 43, 3161 (1965); 44, 1558 (1966).

5. J. G. Powles, A. Hartland, and J. A. Kail, J. Polym. Sci., 55, 361 (1961).

6. J. G. Powles and A. Hartland, Nature, 186, 26 (1960).

7. G. Berry and T. G. Fox, Advan. Polym. Sci., 5, 261, (1968).

8. C. L. Lee, K. E. Polmanter, and E. G. Ring, J. Polym. Sci., Part A-2, 8, 1909 (1970).

9. J. E. Tanner, Kang-Jen Liu, and J.E. Anderson, Macromolecules, 4, 586 (1971).

10. D. K. Green and J. G. Powles, Proc. Phys. Soc. (London), 85, 87 (1965).

11. E. R. Andrew and R. G. Eades, Proc. Roy. Soc. (London), Ser. A, 218, 537 (1953).

12. E. R. Andrew, J. Chem. Phys., 18, 607 (1950).

13. F. A. Bovey, ibid., 32, 1877 (1960).

14. A. Odajima, J. Phys. Soc. Japan, 14, 308 (1959).

15. P. E. Rouse, J. Chem. Phys. 21, 1272 (1953).

16. V. A. Kargin and G. L. Slonimskii, Zh. Fiz. Khim., 23, 563 (1949).

17. T. N. Khazanovich, Vysokomol Soedin., 5, 112 (1963).

18. R. Chûjô, K. Aoki, S. Sato, and E. Nagai, J. Polym. Sci. Part B, 1, 501 (1963).

19. Kang-Jen Liu and R. Ullman, Macromolecules, 2, 525 (1969).

20. D. E. Woessner, J. Chem. Phys., 36, 1 (1962).

21. C. Cunibert, J. Polym. Sci., Part A-2, 8, 2051
(1970).

22. H. A. Resing, J. Chem. Phys., 43, 669 (1965).

23. J.E. Anderson and Kang-Jen Liu, ibid., 49, 2850 (1968).

24. A. J. Barry, J. Appl. Phys., 17, 1020 (1946).

25. J.E. Anderson, Kang-Jen Liu, and Robert Ullman, Discuss. Faraday Soc., 49, 257 (1970).

26. P. J. Flory and J. A. Semlyen, J. Amer. Chem. Soc., 88, 3209 (1966).

27. P. J. Flory, "Statistical Mechanics of Chain Molecules,"' John Wiley and Sons, Inc., New York, N.Y., 1969, Chapt. 5.

28. E.H. Aggarwal and S.H. Bauer, J. Chem. Phys., 42 (1950).

29. P. J. Flory, V. Crescenzi, and J. E. Mark, J. Amer. Chem. Soc., 86, 146 (1964).

30. J. G. Powles and A. Hartland, Proc. Phys. Soc. (London), 75, 617 (1960).

31. Kang-Jen Liu, Macromolecules, 1, 213 (1968).

32. Kang-Jen Liu, J. Polym. Sci., Part A-2, 5, 1199, 1209 (1967).

33. M. J. Newing, Trans. Faraday Soc., 46, 613 (1950).

34. A. Muramoto, Polymer J., 1, 450 (1970).

35. P. Debye, B. Chu, and D. Woermann, J. Chem. Phys., 36, 1803 (1962).

36. T. G. Fox, B. Gratch, and S. Loshck in "Rheology," Vol. 1, F. Eirich, Ed., Academic Press, New York, N.Y., 1956, Chapt. 12.

37. J. E. Anderson, Kang-Jen Liu, and R. Ullman, Discuss. Faraday Soc., 49, 257 (1970).

38. N. Bloembergen, E. M. Purcell, and R. V. Pound, Phys. Rev., 73, 679 (1948). 